\title{
A piezoelectric quad-morph actuated suspension for hard disk drives
}

\author{
Gih Keong Lau ${ }^{1}$ and Hejun Du${ }^{2}$ \\ ${ }^{1}$ Department of Precision and Microsystems Engineering, Faculty of 3ME, \\ Delft University of Technology, Mekelweg 2, 2628 CD Delft, The Netherlands \\ ${ }^{2}$ School of Mechanical and Aerospace Engineering, Nanyang Technological University, \\ Nanyang Avenue, 639798, Singapore \\ E-mail: g.lau@3me.tudelft.nl
}

Received 2 May 2006, in final form 15 June 2006

Published 28 July 2006

Online at stacks.iop.org/JMM/16/1833

\begin{abstract}
This paper presents a new milli-actuator using a piezoelectric quad-morph for fine head positioning in hard disk drives (HDDs). The newly proposed piezoelectric quad-morph is configured to produce a diagonal actuation, driving a parallel mechanism embodied into a suspension base plate into a lateral stroke. Finite element modeling shows the actuated suspension achieves a high sway frequency of $13 \mathrm{kHz}$ and an adequate stroke. A parametric study shows that extending the lengths of the quad-morph and the parallel beams can improve both the sway frequency and the lateral stroke. The actuated suspension can also be reinforced with ribs for enhancing shock resistance and dynamic performance, yet without compromising lateral stroke generation. A prototype of the new design is fabricated and manually assembled using a bulk piezoelectric plate and a photo-chemically machined $0.1 \mathrm{~mm}$ thick stainless steel load beam. The driving principle of the quad-morph is tested and proven a viable and economical method to accomplish fine head positioning.
\end{abstract}

(Some figures in this article are in colour only in the electronic version)

\section{Introduction}

Single-stage actuation of a rotary arm using a voice coil motor has limited capability and servo bandwidth to accomplish fine head positioning for hard disk drives (HDDs). Hence, secondary-stage microactuators were developed to assist the prime actuator. Among the various reported designs, microactuators on the slider and the head levels offer excellent dynamic performance but cost more for requiring miniaturization. In contrast, milli-actuators on the suspension level could offer good performance at low fabrication and assembly cost. As the actuated suspensions are easy to manufacture, they are expected to be adopted in HDDs sooner than other competitive technologies [1].

Actuated suspensions come in various design configurations. Most of them are driven on a push-pull principle with two actuating elements, one moving forwards and the other moving backwards, swinging a magnet head at the tip of the HDD suspension at a magnified lateral stroke.
The actuating elements are often made of piezoelectric plates, which move in either extensional mode [2] or shear mode [3-5]. The piezoelectric extenders are more frequently used than the shear movers because they are easier to manufacture at a lower polarization voltage [6]. Characteristics and design variations of the piezoelectric push-pull actuation are well discussed in the literature. Surveys of them can be found in references like [1-5, 7, 8].

Besides the fabrication cost, the reliability of the actuated suspensions is also a concern [9]. The piezoelectric extenders, mounted over intricate hinges of a movable suspension, are susceptible to more external loading and consequent damages, such as cracks [10]. So far, two methods have been proposed to reduce the damages to piezoelectric extenders. The first method adopted stiffened hinges, for example, the $\phi$-shaped base-plate hinges as proposed by Tokuyama et al [11]. The second method proposed the use of a stiffer piezoelectric extender to maintain structural integrity. For example, multilayered extenders consisting of many thin layers were 


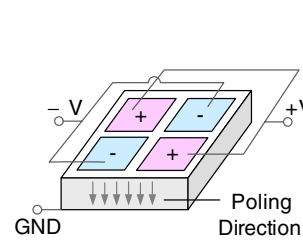

(a)

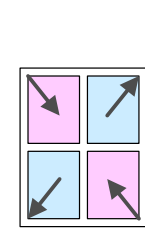

(b)

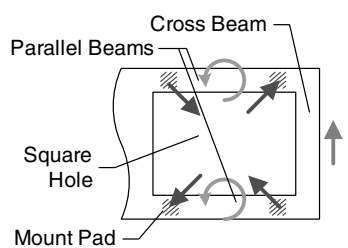

(c)
Figure 1. Actuation using a piezoelectric quad-morph: $(a)$ voltage polarity and poling direction of the piezoelectric plate;

(b) contraction and expansion along two diagonals of the

piezoelectric plate; $(c)$ diagonal actuation forces exerted to a parallel mechanism, producing an upward lateral motion.

adopted for this purpose, as well as achieving a large stroke at a low voltage $[12,13]$. In another example, piezoelectric shearmovers stacked on the base plate were adopted to improve shock resistance of the actuated suspension [4].

This paper proposes a newly-designed piezoelectric quadmorph to drive a HDD suspension, in order to meet the ever demanding requirements for reliability, good dynamic performance and low cost. The quad-morph produces a diagonal actuation on parallel flexures of the suspension, in comparison with a conventional push-pull actuation on pivotal flexures. This is one of two newly proposed suspension designs with parallel flexures, besides the inline actuated suspension [14]. The driving concept of the piezoelectric quad-morph was briefly presented by the same authors at INTERMAG 2005 [15]. In this paper, the new quadmorph design will be further elaborated with finite element modeling, parametric study, and discussions on fabrication and experimental testing.

\section{Actuator design}

The piezoelectric quad-morph is a monolithic plate consisting of four split electrode pads on top and an integral electrode on bottom (see figure $1(a)$ ). It is poled across the thickness like a normal piezoelectric extender, but it is electrically driven in a different configuration. In operation, the diagonal pair of top electrodes is charged with a positive voltage while the other pair is charged with a negative voltage. At such polarity combination of driving voltages, the quad-morph extends along one diagonal while contracting along the other diagonal (see figure $1(b)$ ). The quad-morph is mounted through its four corners onto a parallel mechanism, which is embodied in a base plate of a load beam. As a result of the diagonal extension and contraction, the quad-morph can move the parallel flexures into a lateral motion, normal to the length of the parallel beams (also termed as parallel flexures, see figure 1(c)).

Figure 2(a) shows the load beam for the new design before mounted with the quad-morph. The load beam consists of an extended base plate, equipped with a pair of parallel beams, and an elongated load beam. A spring region of reduced thickness is located between the base plate and the elongated portion of load beam. Four bonding pads, onto which the quad-morph is mounted, are located at the pair of parallel beams. An assembly of the quad-morph and the suspension is shown in figure $2(b)$.

The pair of parallel beams is designed to be $0.5 \mathrm{~mm}$ wide. They are designed with or without ribs. The ribbed designs,

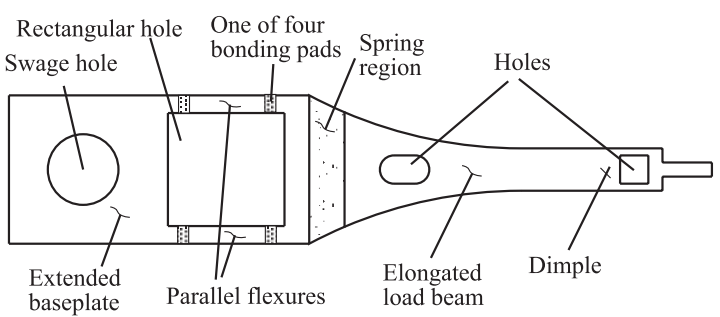

(a)

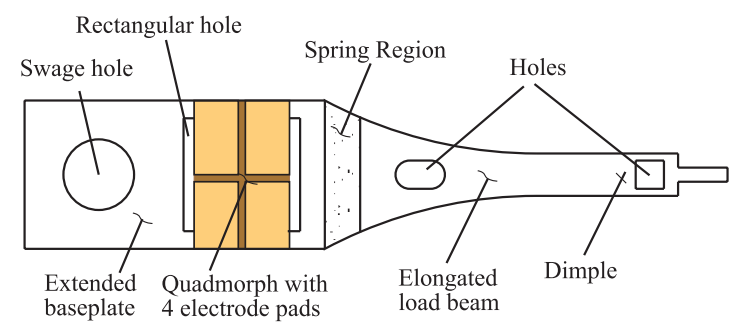

(b)

Figure 2. A new design of actuated suspension: (a) a bare suspension showing two parallel flexures over the extended base plate; $(b)$ assembly of the actuated suspension with quad-morph mounted.

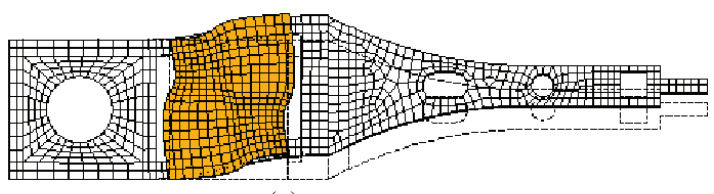

(a)

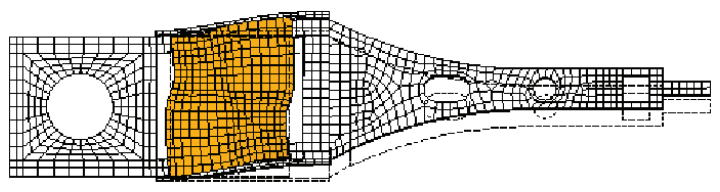

(b)

Figure 3. Deformed shapes (in exaggerated scale) for actuated suspensions, showing diagonal expansion and contraction of piezoelectric quad-morphs: $(a)$ without ribs, $(b)$ with ribs on parallel beams.

with $0.1 \mathrm{~mm}$ or $0.2 \mathrm{~mm}$ high side ribs, can better absorb external loading and prevent the piezoelectric element they carry from being damaged. In addition, the parallel beams are structurally held by the spanning quad-morph when the quad-morph is mounted. Hence, the root of the load beam, at which the parallel beams and the quad-morph are located, is effectively stiffened. In particularly, the lateral and torsional stiffness is enhanced. In comparison, the suspensions for a push-pull design have very intricate hinges of $0.2 \mathrm{~mm}$ wide and they are compromised in both lateral and vertical stiffnesses, leading to higher loading to the piezoelectric plates mounted on them $[14,15]$.

\section{Numerical simulation}

\subsection{Modeling}

Finite element simulation was performed to predict and verify the performance of the newly designed actuated suspension. Figure 3 shows two finite element models of quad-morph-driven suspensions: a planar suspension without 
Table 1. Properties used for piezoelectric ceramics [16].

\begin{tabular}{lr}
\hline Piezoelectric constant & \\
$d_{31}$ & $-340 \times 10^{-12}\left(\mathrm{~m} \mathrm{~V}^{-1}\right)$ \\
$d_{33}$ & $645 \times 10^{-12}\left(\mathrm{~m} \mathrm{~V}^{-1}\right)$ \\
Elastic modulus & \\
$\mathrm{C}_{11}^{\mathrm{E}}$ & $12 \times 10^{10}\left(\mathrm{~N} \mathrm{~m}^{-2}\right)$ \\
$\mathrm{C}_{12}^{\mathrm{E}}=\mathrm{C}_{13}^{\mathrm{E}}$ & $7.7 \times 10^{10}\left(\mathrm{~N} \mathrm{~m}^{-2}\right)$ \\
$\mathrm{C}_{33}^{\mathrm{E}}$ & $11.4 \times 10^{10}\left(\mathrm{~N} \mathrm{~m}^{-2}\right)$ \\
$\mathrm{C}_{55}^{\mathrm{E}}$ & $2.1 \times 10^{10}\left(\mathrm{~N} \mathrm{~m}^{-2}\right)$ \\
Poisson's ratio $\sigma$ & 0.37 \\
Density, $\rho$ & $7800\left(\mathrm{~kg} \mathrm{~m}^{-3}\right)$ \\
\hline
\end{tabular}

Table 2. Properties used for stainless steel.

\begin{tabular}{ll}
\hline Young's modulus & $193.0 \mathrm{GPa}$ \\
Poisson's ratio & 0.30 \\
Density & $8030 \mathrm{~kg} \mathrm{~m}^{-3}$ \\
\hline
\end{tabular}

Table 3. Properties used for cured epoxy [17].

\begin{tabular}{ll}
\hline Young's modulus & $1.3 \mathrm{GPa}$ \\
Poisson's ratio & 0.36 \\
Density & $2120 \mathrm{~kg} \mathrm{~m}^{-3}$ \\
\hline
\end{tabular}

ribs (figure 3(a)) and a rib-reinforced suspension (figure 3(b)). Both static and dynamic analyses were performed to determine performance characteristics of the actuated suspensions. The simulation was done using a commercial finite element software package, ANSYS. The finite element models were constructed using SOLID45 elements for modeling stainless steel suspensions and cured epoxy adhesive, and SOLID5 elements for modeling the piezoelectric materials. The material properties used in the modeling are given in tables $1-3$.

These designs are based on the same planar profile of a load beam, except the difference in rib height. The common dimensions are as follows. The load beam has a thickness of $0.1 \mathrm{~mm}$, an extended base plate of $4.2 \mathrm{~mm}$ wide and $8.0 \mathrm{~mm}$ long, a dimple to swage hole length of $14.5 \mathrm{~mm}$ and a halfetched spring region of $1 \mathrm{~mm}$ long and $0.05 \mathrm{~mm}$ thick. The elongated load beam is $1.2 \mathrm{~mm}$ wide at its distal end and it is equipped with an end lift tip for the purpose of load-unload. The actuated load beams carry a $3.6 \mathrm{~mm}$ long, $4.2 \mathrm{~mm}$ wide and $0.1 \mathrm{~mm}$ thick piezoelectric quad-morph, which has four split electrodes on one side but an integral electrode on the other side. The bonding area for each pad is set to be $0.5 \mathrm{~mm}$ wide and $0.35 \mathrm{~mm}$ long.

A clamped-free boundary condition is imposed on the actuated suspensions in all simulations done. This describes the condition of the suspension assembly before being mounted to a disk drive. Four weld points, around the swage hole, and the swage hole itself are fully fixed while other portions of the load beam are free to move. In addition to the boundary condition, several loading conditions are imposed in different analyses. In the case of a static analysis, a driving voltage of $30 \mathrm{~V}$ is applied to a pair of diagonal electrodes while a negative voltage of the same magnitude is applied to the other diagonal pair of the quad-morph. In the case of a harmonic analysis, harmonic driving voltages over a range of frequencies are imposed on the whole assemblies for predicting frequency responses. In the case of a quasi-shock analysis, an out-ofplane acceleration of $100 \mathrm{G}$ is imposed on the assemblies.

\subsection{Results and discussion}

Table 4 shows simulated performance of three actuated suspensions, with or without ribs, carrying a $3.6 \mathrm{~mm}$ long quad-morph on one side of the load beams. The three actuated suspensions achieve a lateral stroke of at least $0.35 \mu \mathrm{m}$. Figure 3 shows the simulated deformed shapes of the quadmorph driven suspensions, one without ribs and one with $0.2 \mathrm{~mm}$ high ribs. It is observed that the elongated suspensions of the two designs translate laterally, together with the parallel mechanisms, when the quad-morph actuates diagonally.

During the in-plane actuation by the piezoelectric quadmorph, the suspensions are simultaneously twisted, producing unintended out-of-plane motion as shown in figure 4 . The finite element simulation predicts that dimple point of the actuated suspensions deflects very slightly when driven at a $30 \mathrm{~V}$. The out-of-plane displacements at the dimple are calculated as $18 \mathrm{~nm}$ and $15 \mathrm{~nm}$ respectively for designs without ribs and with $0.2 \mathrm{~mm}$ high ribs. Therefore, when mounted on a disk plate, the actuated suspensions of small vertical spring rate (several tens $\mathrm{N} \mathrm{m}^{-1}$ ) have a low risk in perturbing the flying height of a slider, which is levitated by an air bearing of relatively high vertical stiffness in the range of several hundreds $\mathrm{kN} \mathrm{m}^{-1}$ [18].

Comparison in table 4 shows that the side ribs on the parallel beams have a slight effect on the lateral stroke. The lateral stroke decreases slightly with increasing rib height, which increases in-plane stiffness. The lateral stroke obtained for the present design is not as high as those obtained for the conventional push-pull designs with pivotal hinges. This is because the proposed actuation is induced by diagonal strokes through parallel beams, rather than lever magnification as for the push-pull design.

The three actuated suspensions are simulated to have high sway frequencies, at least $13.52 \mathrm{kHz}$. Increasing rib height slightly increases the torsional and the sway resonant frequencies. However, the increase in rib height significantly raises the spring rate of the suspensions and reduces the induced maximum stress in the piezoelectric element. Figure 5 shows the stress contour on the actuated suspensions, with or without ribs, when subjected to a static $100 \mathrm{G}$ out-ofplane acceleration. It is observed that the steel suspension undergoes the highest stress at the root of the parallel flexure, absorbing most of the impact. However, the quad-morphs on the suspensions are still subjected to some impact, at a fraction of those maxima on the suspensions. Among the designs listed in table 4, the piezoelectric quad-morph on the suspension with $0.2 \mathrm{~mm}$ high rib is subjected to the least acceleration-induced stress.

A suspension carrying the quad-morph only on one side, like those presented in table 4, is subjected to mass unbalance. This is because the one-sided quad-morph does not lie on the neutral plane as the suspension. When actuated by the quadmorph, the suspension is twisted and consequently suffers from a lower torsional frequency. Figure 6 shows self-driven frequency responses for the actuated suspensions with and without ribs. The designs carrying a quad-morph on one side, with or without ribs, undergo the first torsional resonance at 

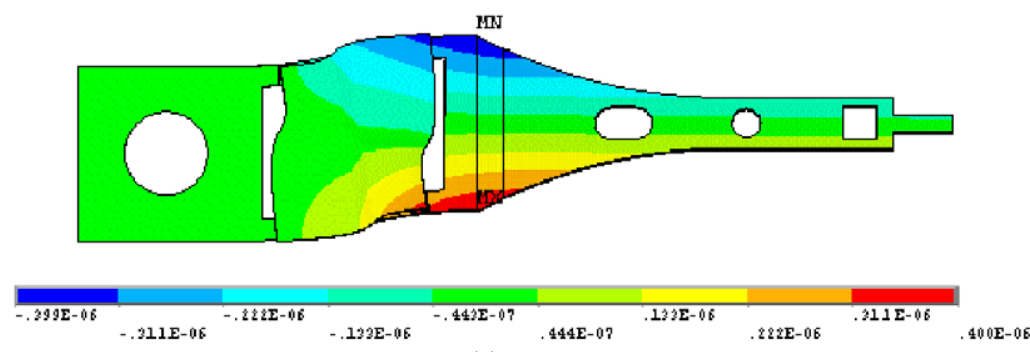

(a)
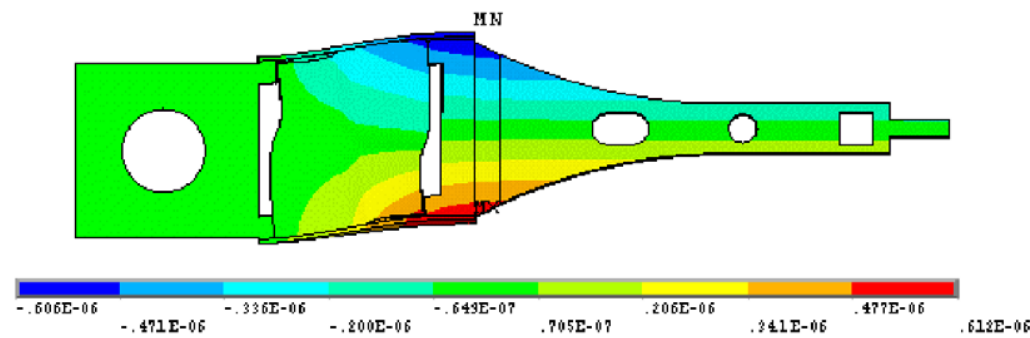

(b)

Figure 4. Contour plot (with color bars in units of $\mathrm{m}$ ) showing un-intended out-of-plane motion produced during the in-plane actuation of the piezoelectric quad-morph): ( $a$ ) on the actuated suspension without side ribs on the parallel flexures and $(b)$ on the actuated suspension with $0.2 \mathrm{~mm}$ high side ribs on the parallel flexures.
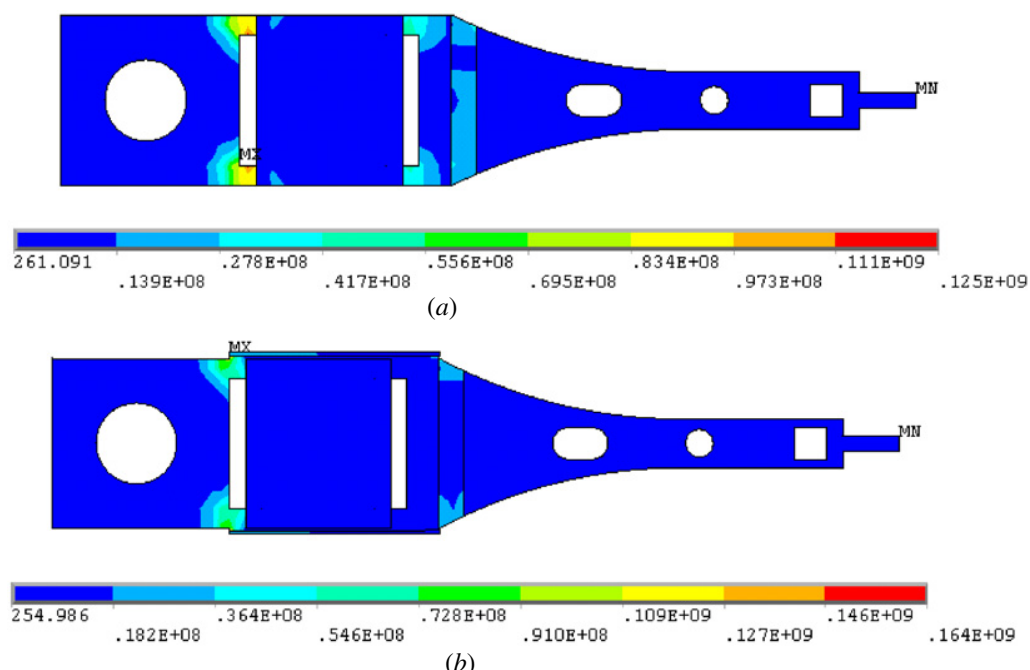

(b)

Figure 5. Stress contours (with color bars in units of $\mathrm{Pa}$ ) of the actuated suspensions under a static out-of-plane acceleration of $100 \mathrm{G}$ : (a) an actuated suspension without ribs, $(b)$ an actuated suspension with $0.2 \mathrm{~mm}$ high ribs.

Table 4. Simulated performance of actuated suspensions using a $3.6 \mathrm{~mm}$ long quad-morph.

\begin{tabular}{lccc}
\hline & \multicolumn{3}{c}{ Actuated suspension } \\
\cline { 2 - 4 } & Without rib & With 0.1 mm ribs & With 0.2 mm ribs \\
\hline Lateral stroke $(\mu \mathrm{m})$ at 30 V & 0.37 & 0.36 & 0.35 \\
Cantilever frequency $(\mathrm{kHz})$ & 0.42 & 0.47 & 0.51 \\
First torsion frequency $(\mathrm{kHz})$ & 4.38 & 4.58 & 4.57 \\
Second torsion frequency $(\mathrm{kHz})$ & 12.09 & 12.68 & 12.87 \\
First sway frequency $(\mathrm{kHz})$ & 13.52 & 13.83 & 14.15 \\
Max. induced stress in PZT & 13.70 & 9.36 & 5.81 \\
at 100 G $(\mathrm{MPa})$ & 37.22 & 47.38 & 54.58 \\
Spring rate $\left(\mathrm{N} \mathrm{m}{ }^{-1}\right)$ & & & \\
\hline
\end{tabular}

around $4.4 \mathrm{kHz}$, as illustrated with vibration spike in a dashed line. However, a rib-less design carrying quad-morphs on both sides does not exhibit undue twisting at the low frequency when self-driven into vibration as shown in a solid line of 1836 figure 6. The improvement of the double-side lapped design is attributed to the thickness symmetry. Despite this, the onesided quad-morph is preferred due to the concern over material cost. 


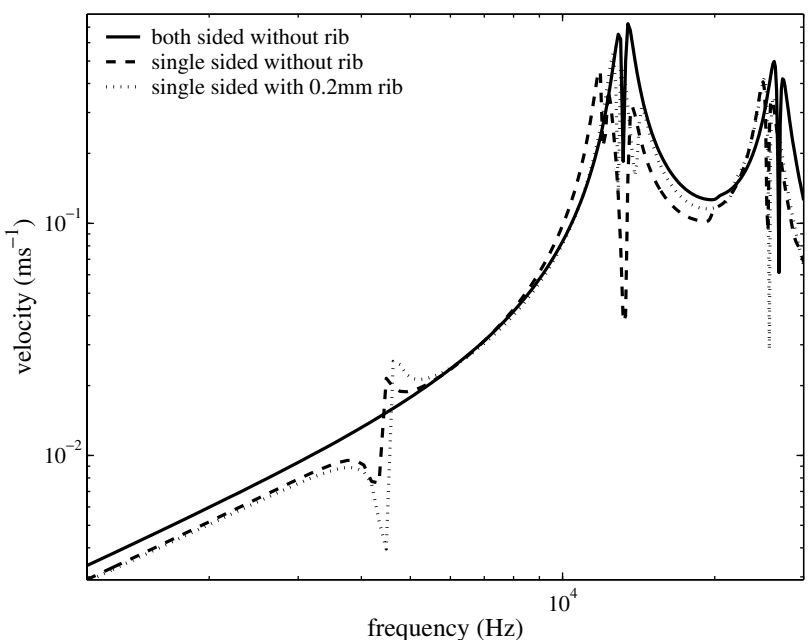

Figure 6. Simulated frequency response for the quad-morph-driven suspensions.

\subsection{Parametric study}

A longer piezoelectric quad-morph could increase the magnitude of diagonal actuation, but it requires more room or lengthened parallel beams for mounting. The longer quadmorph and the longer parallel beams are expected to have large influence on both static and dynamic performances of the actuated suspension. In this subsection, a parametric study will be conducted to investigate the length effects of the piezoelectric quad-morph and the parallel beams on the suspension performances.

Figure 7 shows performance changes with respect to the length of the piezoelectric quad-morphs, which is proportional to that of the carriage parallel beams. The longer piezoelectric quad-morph leads to a larger stroke and added mass and stiffness. But the proportionally longer parallel beams, which are formed by cutting a hole into a base plate of constant length, result in mass and stiffness reduction. Figure 7(a) shows that the spring rate of the suspension assembly increases with increasing quad-morph length, as a result of the stiff
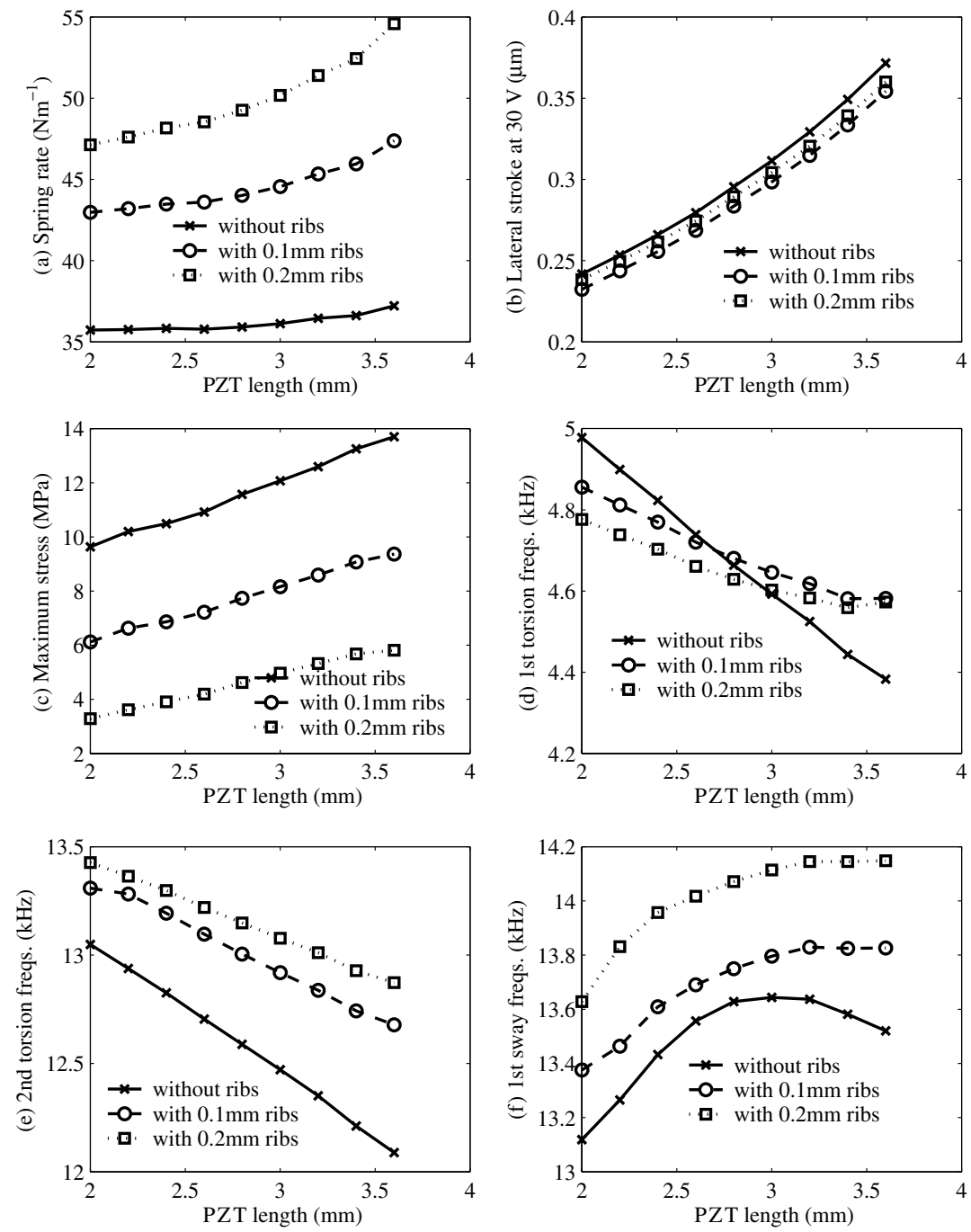

Figure 7. Trends of response with respect to PZT plate length at the constant length of the extended base plate (9.6 mm): $(a)$ spring rate $\left(\mathrm{N} \mathrm{m}^{-1}\right) ;(b)$ lateral stroke $(\mu \mathrm{m})$ at $30 \mathrm{~V} ;(c)$ maximum stress induced in the PZT plate under an out-of-plane acceleration of $100 \mathrm{G} ;(d)$ first torsional frequency $(\mathrm{kHz}) ;(e)$ second torsional frequency $(\mathrm{kHz})$ and $(\mathrm{f})$ first sway frequency $(\mathrm{kHz})$. 


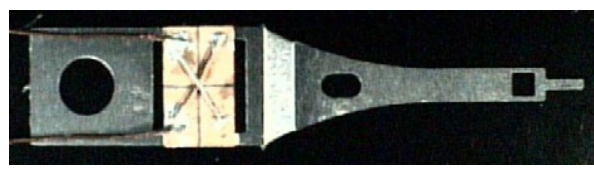

(a)

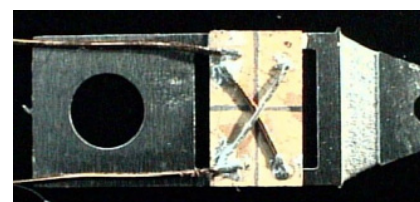

(b)

Figure 8. Prototype of the quad-morph actuated suspension: (a) full view, $(b)$ zoomed view.

quad-morph compensating for the reduced stiffness of the elongated beams. However, the lateral stroke increases with the increasing length of quad-morph as a result of the combined length effects (see figure $7(b)$ ). As both the quad-morph and parallel beams are elongated, more maximum stress is induced in the piezoelectric quad-morph under a static acceleration of $100 \mathrm{G}$ (see figure $7(c)$ ).

Figures $7(d)-(f)$ show that the quad-morph length has a slight effect on the suspension dynamics. It is observed from figures $7(d)-(e)$ that both the first and the second torsional frequencies decrease with increasing quad-morph length at small margins. The decrease in torsional frequencies is due to added un-balanced mass and reduced beam stiffness. Figure $7(f)$ shows that the first sway frequency does not decrease with increasing quad-morph length. In the case of rib-reinforced suspensions, the sway frequencies increase slightly with the length at a decreasing rate. In the case of rib-less suspension, the sway frequency appears parabolic with respect to the increasing length. The change in sway frequency with respect to the length shows that the quad-morph stiffens in-plane the elongated parallel beams to a certain extent.

An interesting finding is observed by comparing the trend of the lateral stroke (figure $7(b)$ ) with that of the sway frequency (figure $7(f)$ ) at increasing quad-morph length. It is found that the lateral stroke does not decrease with increasing the sway frequency as both the piezoelectric quad-morph and parallel beams are elongated. This is quite different from the trends for most push-pull actuated suspensions, which have the decreasing lateral stroke at the increasing sway frequency. The difference between the two trends clearly shows the significant influence of actuation mechanisms on the suspension performances, both static and dynamic.

Also shown in figure 7 is the effect of ribs on both static and dynamic responses of the actuated suspensions. It is observed the ribs reinforce vertical stiffness, leading to an increasing spring rate (figure $7(a)$ ) but decreasing maximum stress induced to the quad-morph (figure $7(c)$ ). However, it hardly affects in-plane stiffness, as shown by the clustering trend lines of the lateral strokes at different rib heights. In addition, the rib height is found to have some influences on the resonant frequencies. The first torsional frequency shows varying trends with respect to the rib height at different quad-morph lengths: it decreases slightly with increasing rib height for the shorter lengths, but it increases slightly with increasing rib height for the longer lengths. The second torsional frequency increases with increasing rib height. On the other hand, the sway frequency is found to increase slightly with increasing rib height.

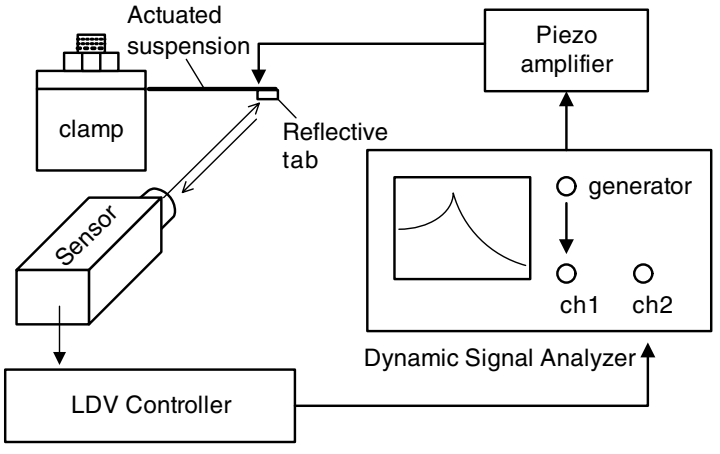

Figure 9. A schematic showing the experiment setup used for measuring the velocity amplitude of a self-driven quad-morph actuated suspension.

\section{Testing}

\subsection{Fabrication}

A piezoelectric quad-morph and a stainless steel suspension are separately processed before they are assembled together. A standard piezoelectric plate has integral electrodes on both sides. It needs a further process to make a quad-morph, which has four separate electrodes on the top. The standard plate is first laminated by dry resist films on the bottom electrode and the four separate top electrodes. A cross slit, which is not masked on the top metallic electrode, is chemically etched using gold and chromium etchants. On the other hand, a suspension without ribs is formed, by photochemical machining a $0.10 \mathrm{~mm}$ thick stainless steel foil. After making the two components, they are assembled manually with the help of tweezers and an optical microscope. The quad-morph is adhesively bonded through its four corners to two parallel beams of the load beam. The adhesive is then thermally cured on a hot plate. In the final step, copper wires are bonded to the piezoelectric quad-morph to form interconnect between the diagonal pads and to form leads out to voltage supplies. The assembled prototype of the actuated suspension without rib is shown in figure 8 .

\subsection{Measurement}

Figure 9 shows a schematic of the experiment setup used to measure the in-plane velocity amplitude of the actuated suspension. In the setup, the suspension is clamped at its base plate using a steel clamp holder. The bottom electrode surface of the plate, together with the load beam, is grounded through the clamp holder while the top diagonal electrode pads are supplied with $30 \mathrm{~V}$ and $-30 \mathrm{~V}$ respectively through the copper 

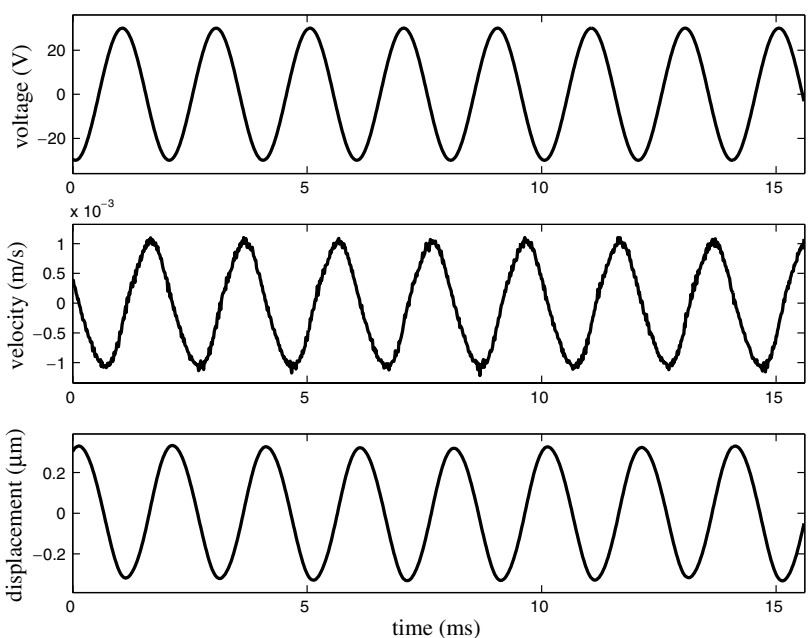

Figure 10. Measured harmonic responses at $500 \mathrm{~Hz}$ for the quad-morph-actuated suspension, showing driving voltages, measured velocity and numerically derived displacement.
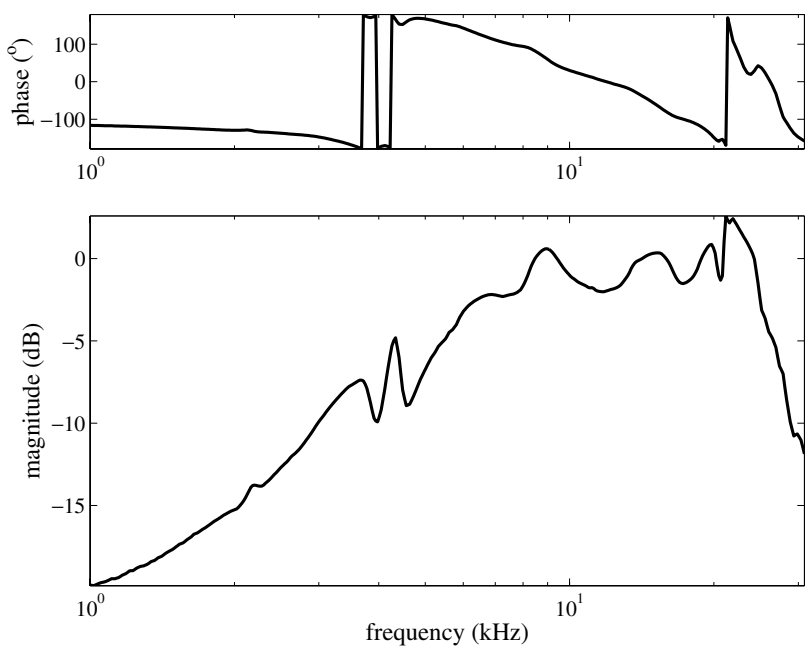

Figure 11. The measured lateral frequency response of the actuated suspension, ranging from $1 \mathrm{kHz}$ to $25 \mathrm{kHz}$.

wires. A reflective tab is adhered to the tip of the suspension. Such a tab is needed because the sidewall of suspension is too thin and too rough to properly reflect a laser light shot for an in-plane velocity measurement. The system used for velocity measurement is a Polytec scanning laser Doppler vibrometer. With the setup, the actuated suspension is self-driven into vibration. The measured sensor signal and the input signal are fed into a dynamic signal analyzer to display the frequency responses during the modal testing.

First, sinusoidal voltages at $500 \mathrm{~Hz}$ are supplied to measure the in-plane velocity of the actuated suspension. Figure 10 shows the sinusoidal input voltage, the measured velocity at the suspension tip, and the numerical derived displacement at the tip. The magnitude of the derived lateral displacement is $0.33 \mu \mathrm{m}$, which is smaller than the predicted static value of $0.37 \mu \mathrm{m}$ in table 4 . The discrepancy may be attributed to dimensional deviation of adhesive thickness, bonding areas and flexural width of parallel beams for suspension.
Figure 11 shows the frequency response of the quadmorph-actuated suspension, sweeping from $1 \mathrm{kHz}$ to $25 \mathrm{kHz}$. The first torsional frequency is measured at around $4 \mathrm{kHz}$, which is close to the simulated one of $4.38 \mathrm{kHz}$. However, vibration peaks of the second torsional and the first sway resonances are not very distinct and lead to poorer identification. They are somewhere around 9-12 kHz. The poor identification and measurement discrepancy may be attributed to the thickness unbalance of the copper wires bonded onto the quad-morph that was not accounted for in the finite element model.

\section{Conclusion}

This paper presented a novel design of a milli-actuator using a piezoelectric quad-morph for driving a pair of parallel beams embedded in a suspension base plate. Numerical studies showed that suspensions driven with this piezoelectric actuator can achieve an adequate lateral stroke and a high sway frequency. Parametric study showed that the lateral stroke is not compromised by the increased sway frequency when both the quad-morph and the parallel beams are elongated. If the parallel beams are reinforced with ribs of $0.1-0.2 \mathrm{~mm}$, shock resistance is tremendously improved, accompanying by slight enhancement of resonant frequencies, yet almost no reduction in lateral stroke. A prototype of the actuated suspension using the quad-morph was experimentally tested and the driving principle was verified.

\section{Acknowledgments}

This project is funded by the Agency of Science, Technology and Research of Singapore through the Center for Mechanics of Micro-Systems (CMMS). The authors would like to acknowledge Dr Guo G X and Dr Ong E H of the Data Storage Institute, Singapore for insightful comments. The authors also would like to thank Dr Zhu H for providing help in chemical etching, and Mr Cheo H L and Mrs Halimatun for laboratory support.

\section{References}

[1] Koganezawa S and Hara T 2001 Development of shear-mode piezoelectric microactuator for precise head positioning Fujitsu Sci. Tech. J. 37 212-9

[2] Takaishi K, Imamura T, Mizoshita Y, Hasegawa S, Ueno T and Yamada T 1996 Microactuator control for disk drive IEEE Trans. Magn. 32 1863-6

[3] Koganezawa S, Uematsu Y, Yamada T, Nakano H, Inoue J and Suzuki T 1998 Shear mode piezoelectric microactuator for magnetic disk drives IEEE Trans. Magn. 34 1910-2

[4] Koganezawa S, Uematsu Y, Yamada T, Nakano H, Inoue J and Suzuki T 1999 Dual-stage actuator system for magnetic disk drives using a shear mode piezoelectric microactuator IEEE Trans. Magn. 35 988-92

[5] Koganezawa S, Hara T, Uematsu Y and Yamada T 2001 Effect of dual-stage actuator on positioning accuracy in $10 \mathrm{k} \mathrm{rpm}$ magnetic disk drives IEEE Trans. Magn. 37 955-8

[6] TRS Ceramics 1999 Piezoelectric Actuators at TRS http://www.trsceramics.com/actuators.html

[7] Niu Y, Guo W, Guo G, Ong E H, Sivadasan K K and Huang T 2000 A PZT micro-actuated suspension for high TPI hard disk servo systems IEEE Trans. Magn. 36 2241-3 
[8] Lau G K and Du H 2005 A piezoelectric micro-actuator with extended base-plate for HDD Microsyst. Technol. 11 598-605

[9] Nakamura S, Naniwa I, Sato K, Yasuna K and Saegusa S 2001 Lifetime prediction method for piggyback PZT actuator IEEE Trans. Magn. 37 940-3

[10] Yeh Y-P, McCaslin M and Ku C-P R 2000 PZT crack detection in suspension-based dual stage actuator IEEE Trans. Magn. 36 2244-6

[11] Tokuyama M, Shimizu T, Masuda H, Nakamura S, Hanya M, Iriuchijima O and Soga J 2001 Development of a phi-shaped actuated suspension for 100-kTPI hard disk drives IEEE Trans. Magn. 37 1884-6

[12] Naniwa I, Nakamura S, Saegusa S and Sato K 1999 Low voltage driven piggy-back actuator of hard disk drives Proc 12th IEEE Int. Conf. on Micro Electro Mechanical Systems 1999 (MEMS '99) pp 49-52
[13] Wang Z H, Zhu W G and Yao X 2002 A d(31) type inplane bending multilayer piezoelectric microactuators - a design concept and its applications Sensors Actuators A $101262-8$

[14] Lau G K and Du H 2006 Inline-actuated suspension for the fine head positioning of HDD J. Micromech. Microeng. 16 1416-23

[15] Du H J, Lau G K and Liu B 2005 Actuated suspensions with enhanced dynamics for hard disk drives IEEE Trans. Magn. 41 2887-9

[16] Fuji Ceramics 1999 The Table for Piezo Ceramic Material Constants (Tokyo: Fuji Ceramic Corporation)

[17] Loctite 1998 Technical Data Sheet for Product 3880 (Dublin, Ireland: Loctite Research, Development and Engineering)

[18] Wada Y, Roppongi T and Sato I 1999 Flying characteristics of shallow step pads slider for low flying IEEE Trans. Magn. 35 2475-7 